\title{
Estruturação do Espaço Farmácia Viva na Universidade Federal de Pernambuco como Estratégia para Formação em Fitoterapia
}

\author{
René Duarte Martins ${ }^{\mathrm{a}^{*}}$, Rogélia Herculano Pinto ${ }^{\mathrm{b}}$, Sueli Moreno Senna ${ }^{\mathrm{b}}$, Ana Wládia \\ Silva de Lima ${ }^{b}$, Carlos Renato de França de Carvalho Mota ${ }^{c}$, Danilo Augusto Ferreira \\ Fontes $^{\mathrm{d}}$, Francisco Amorim de Barros ${ }^{\mathrm{e}}$, Rafael Matos Ximenes ${ }^{\mathrm{f}}$ \\ ${ }^{a}$ Núcleo de Saúde Coletiva, Centro Acadêmico de Vitória (CAV), Universidade Federal de Pernambuco, Vitória de \\ Santo Antão, PE, Brasil \\ ${ }^{\mathrm{b}}$ Núcleo de Enfermagem, Centro Acadêmico de Vitória (CAV), Universidade Federal de Pernambuco, Vitória de \\ Santo Antão, PE, Brasil \\ ${ }^{\mathrm{c}}$ Centro Acadêmico do Agreste (CAA), Universidade Federal de Pernambuco, Caruaru, PE, Brasil \\ ${ }^{\mathrm{d}}$ Centro Acadêmico de Vitória (CAV), Universidade Federal de Pernambuco, Vitória de Santo Antão, PE, Brasil \\ ${ }^{\mathrm{e}}$ Hospital das Clínicas (HC-UFPE), Universidade Federal de Pernambuco, Recife, PE, Brasil \\ ${ }^{\mathrm{f}}$ Departamento de Antibióticos, Centro de Biociências, Universidade Federal de Pernambuco, Recife, PE, Brasil
}

Histórico do Artigo

Recebido em:

$15 / 11 / 2017$

Aceito em:

$30 / 03 / 2018$

Palavras-Chave:

Horto de plantas medicinais; práticas integrativas e complementares; disciplinas de graduação; projetos de extensão.

Key-Words:

Garden of medicinal plants; integrative and complementary practices; academic disciplines for graduate; extension projects.

\section{RESUMO}

Um horto didático de plantas medicinais visa recuperar saberes populares e tradicionais, discuti-los cientificamente para contribuir com a construção do conhecimento, divulgação de informações e formação em fitoterapia. Este trabalho objetiva relatar as experiências didáticas vivenciadas no Espaço Farmácia Viva do CAV/UFPE. O marco teórico para estruturação desse espaço ocorreu no ano de 2009, por intermédio do edital de extensão PROEXT 2009 MEC/SESu, cujo financiamento permitiu aquisição de materiais e desenvolvimento do programa "Farmácia Viva: Cultivando Saúde", primeira ação de extensão em Práticas Integrativas e Complementares (PICs), desenvolvida no Município de Vitória de Santo Antão, com a presença da Universidade Federal de Pernambuco. Desde então, o referido programa gestou iniciativas que ampliaram a oferta acerca da discussão sobre PICs no âmbito acadêmico e municipal, com marcante presença de diálogo entre Universidade e Serviços de Atenção Básica ofertados no município. Assim, ao longo da existência dessa iniciativa, a implantação do horto ocorreu entre os anos de 2011/2013, por meio de mudas certificadas, oriundas do Horto de Plantas Medicinais Francisco José de Abreu Matos da UFC e do Núcleo de Fitoterapia da Coordenadoria de Assistência Farmacêutica da Secretaria de Saúde do Estado do Ceará, onde a coordenação do projeto teve oportunidade de realizar treinamento em 2010. O contato com as plantas medicinais e a relação com os sentidos humanos promovidos por essas interações, por meio de cursos, oficinas, projetos de pesquisa/extensão e disciplinas acadêmicas para graduandos e residentes, despertaram vivências fundamentais na formação de profissionais sensíveis e reflexivos acerca do uso racional de plantas medicinais e de fitoterápicos.

Structure of the "Espaço Farmácia Viva" at the Federal University of Pernambuco as a Strategy for Formation in Phytotherapy

\section{ABSTRACT}

A didactic garden of medicinal plants aims to recover popular and traditional knowledge, scientifically discuss them to contribute to the construction of knowledge, dissemination of information, and training on phytotherapy. The objective of this work is to report the didactic experiences lived in the "Espaço Farmácia Viva" of the CAV/UFPE. Theoretical framework for the structuring of this space took place in 2009, through a call for extension projects PROEXT 2009 - MEC/SESu, of which the funding line allowed the acquisition of materials and development of the "Living Pharmacy: Cultivating Health" program, the first extension action in Integrative and Complementary Practices (ICPs) developed in Vitória de Santo Antão, with the presence of UFPE. Since then, the program has promoted initiatives that have broadened the offer on the discussion of ICPs in both academic and municipal spheres, with a significant presence of dialogue between University and Basic Assistance Services. Thus, throughout the existence of this initiative, the establishment of the garden occurred between 2011/2013, through certified seedlings from the Francisco José de Abreu Matos Garden of the UFC and Nucleus of Phytotherapy in the Pharmaceutical Assistance Coordination of the Health

\footnotetext{
${ }^{*}$ Autor correspondente: reneduarte.ufpe@gmail.com (R. D. Martins)
} 
Department in the State of Ceará, where the coordination of the project had the opportunity to carry out training sessions in 2010 . The contact with medicinal plants and the relation with human senses promoted by this interaction through courses, workshops, research and extension projects, and academic disciplines for undergraduate students and residents, have awakened fundamental experiences in the formation of sensitive and reflective professionals about the rational use of medicinal plants and phytotherapics.

\section{Introdução}

O conhecimento e uso terapêutico de plantas medicinais se confunde com a própria existência do homem e sua relação com a natureza (1), encontrando, na atualidade, a Estratégia de saúde da Família (ESF) como campo fértil para seu uso e reconhecimento no Sistema Único de Saúde (SUS) (2).

Dentre as diretrizes da Política Nacional de Plantas Medicinais e Fitoterápicos, encontram-se "Incentivar a formação e a capacitação de recursos humanos para o desenvolvimento de pesquisas, tecnologias e inovação em plantas medicinais $e$ fitoterápicos" e "Promover a Formação técnico -científica e a capacitação no setor de plantas medicinais e fitoterápicos" (3). Nesse contexto, a estruturação de espaços de formação permanente, para interlocução entre saberes que garantam o reconhecimento de práticas populares e o fomento à pesquisa, com manejo sustentável da biodiversidade (3), encontram, nos hortos didáticos de plantas medicinais, ambientes propícios para discussões informativas e formativas sobre fitoterapia.

Segundo Nunes (2002) (4), O Horto didático de Plantas Bioativas possui por objetivo resgate, conscientização e incentivo à preservação do meio ambiente. Para Lopes e Link (2011) (5), "O horto didático é uma unidade de referência de plantas bioativas catalogadas de acordo com a biodiversidade local, que serve para orientar quanto à classificação, identificação e uso", promovendo a conexão entre o saber científico e o saber popular, valorizando esse etnoconhecimento (6).

Em pesquisa realizada em Pacatuba/SE, no ano de 2011, observaram-se equívocos na manipulação de plantas medicinais que podem afetar a efetividade das práticas em fitoterapia e expor os usuários a riscos. Nesse estudo, um plano de intervenção envolvendo oficinas de formação entre usuários de plantas medicinais e profissionais de saúde gerou uma sugestão de protocolo para intervenções em comunidades, com enfoque na formação e informação como estratégias do uso racional de plantas medicinais e manejo sustentável da biodiversidade (7).

A implantação do Espaço Farmácia Viva no Centro Acadêmico de Vitória de Santo Antão/UFPE representou a ampliação de possibilidades para a discussão sobre uso de plantas medicinais e a necessidade da abordagem sobre esse conhecimento entre estudantes de graduação, residentes, profissionais de saúde do município e usuários do SUS. Diversos projetos de extensão, pesquisa e disciplinas de graduação emergiram como referência desse espaço. Este trabalho objetivou relatar a experiência de implementação do Espaço Farmácia Viva no CAV/UFPE, como recurso didáticopedagógico para a formação de profissionais de saúde sensíveis a trabalhar com práticas integrativas e complementares, em especial com a fitoterapia.

\section{Materiais e Métodos}

\subsection{Caracterização do Local}

O município de Vitória de Santo Antão localiza-se a 55km da cidade do Recife, capital do Estado de Pernambuco. Possui 335,941 Km² e população estimada para 2017 de 137.578 mil habitantes (8). 
A Universidade Federal de Pernambuco iniciou suas atividades nesta região, em 21 de Agosto de 2006, com a implantação do Centro Acadêmico da Vitória (CAV), inicialmente, com os cursos de graduação em Enfermagem, Nutrição e Licenciatura em Ciências Biológicas, oportunamente seguidos pelo Bacharelado e Licenciatura em Educação Física e, mais recentemente, pelo Bacharelado em Saúde Coletiva.

\subsection{Espaço Farmácia Viva}

Estrutura Física: O espaço encontra-se composto pelo Horto Didático de Plantas Medicinais, Espaço de Triagem de Plantas Medicinais, Oficina de Manipulação e Sala de Formação. O horto possui $134,4 \mathrm{~m}^{2}$ e comporta 15 canteiros para cultivos de espécies medicinais utilizadas nas discussões de projetos e aulas. A área coberta do Espaço Farmácia Viva é composta por (A) Espaço de Triagem de plantas medicinais, com, aproximadamente, $18 \mathrm{~m}^{2}$, composto por bancadas e ponto de água; (B) Sala de Formação, com, aproximadamente, $20 \mathrm{~m}^{2}$, onde há infraestrutura representada por material audiovisual, cadeiras e mesas redondas, para discussões de projetos/ações e aulas expositivas; (C) Laboratório de Manipulação, com aproximadamente $15 \mathrm{~m}^{2}$, o qual conta com bancadas, ponto de água e equipamentos para realização de aulas práticas e manipulação de fitoterápicos utilizados em projetos.

Finalidade: Desenvolver atividades de Ensino, Pesquisa e Extensão com abordagem direcionada para a formação em Fitoterapia e uso/manejo sustentável da biodiversidade, com enfoque nas discussões sobre plantas locais/regionais e recursos para o SUS.

Projetos Desenvolvidos: O espaço encontra-se disponível para a realização de atividades diversas, cujos objetivos estejam em consonância com as missões formativa e informativa. Desde a estruturação do espaço, foi desenvolvida ampla diversidade de projetos que apresentaram aprovações institucional e/ou por órgãos de fomento, cujas descrições ilustrarão o resultado deste relato (Quadro 2).

Espécies Cultivadas: As espécies certificadas cultivadas originaram-se, em sua maioria, do Horto de Plantas Medicinais Francisco José de Abreu Matos, do Projeto Farmácias Vivas da Universidade Federal do Ceará - UFC e do Núcleo de Fitoterapia da Coordenadoria de Assistência Farmacêutica da Secretaria da Saúde do Estado do Ceará - NUFITO. As demais espécies foram reconhecidas botanicamente e as exsicatas referentes encontram-se depositadas no Instituto Agronômico de Pernambuco (IPA). Algumas espécies presentes no espaço foram trazidas por voluntários ou emergem espontaneamente, subsidiando discussões sobre características daninhas de algumas ervas medicinais, extrativismo e conhecimento popular.

Dados Apresentados: Os dados apresentados nos resultados deste relato encontramse registrados nas plataformas Sigproj/UFPE (Sistema de Informação e Gestão de Projetos) e Sig@ UFPE (Sistema de Informação e Gestão Acadêmica).

\section{Resultados e Discussão}

No ano de 2009, a aprovação do Programa Farmácia Viva: Cultivando Saúde no Edital n 06 - Programa de Extensão Universitária - PROEXT 2009/Ministério da Educação, por intermédio da SESu/DIFES, deu início ao espaço (9). Essa aprovação financiou aquisição de equipamentos e material de consumo para início das atividades na modalidade de extensão, cujo objetivo foi construir canteiros de plantas medicinais e condimentares (nativas e não nativas), com ênfase na troca de experiências com a população, visando adequar a Farmácia Viva à realidade da população local, o que foi reforçado através da organização de oficinas nas Unidades Básicas de Saúde/Estratégia 
de Saúde da Família (UBS/ESF) e das Escolas do Estado e Município, com o intuito de orientar o cultivo e a manipulação correta de espécies medicinais e condimentares.

$\mathrm{O}$ horto de plantas medicinais do CAV/UFPE possui plantas medicinais certificadas que cumprem requisitos para utilização em distúrbios de baixa complexidade com segurança, por meio de formulações extemporâneas ou produção de fitoterápicos, perfazendo um total de 30 espécies, conforme demonstrado no Quadro 1. As espécies presentes no horto encontram-se distribuídas em 18 famílias, com predomínio da família Lamiaceae (33\%).

A família Lamiaceae possui 236 gêneros e entre 6.900 a 7.200 espécies. Estão presentes nessa família espécies de porte predominantemente herbáceo e arbustivo, produtoras de óleos essenciais, como mentol, carvacrol, timol, limoneno, cineol e carvona, com atividades biológicas variadas, como antioxidantes, fungicidas, bactericidas, inseticida, imunomoduladora, antialérgicas, antidiabéticas e adaptógenas, além de amplo uso condimentar e na indústria alimentícia (10-12).

Quadro 1: Espécies De Plantas Medicinais Presentes no Horto do Espaço Farmácia Viva do Centro Acadêmico de Vitória da Universidade Federal de Pernambuco

\begin{tabular}{|c|c|c|}
\hline $\begin{array}{l}\text { Planta Medicinal } \\
\text { (Nome Popular) }\end{array}$ & $\begin{array}{c}\text { Espécie } \\
\text { (Nome Científico) }\end{array}$ & Indicações \\
\hline Alecrim & Rosmarinus officinalis L. (Lamiaceae) & Condimentar, Digestória \\
\hline Alecrim Pimenta & $\begin{array}{l}\text { Lippia origanoides Kunth (sin. Lippia } \\
\text { sidoides Cham.) (Verbenaceae) }\end{array}$ & $\begin{array}{l}\text { Antisséptica, } \\
\text { Antimicrobiana }\end{array}$ \\
\hline Alfavaca & Ocimum gratissimum L. (Lamiaceae) & $\begin{array}{l}\text { Antisséptica das Vias } \\
\text { Aéreas, Expectorante, } \\
\text { Enxaguatório Bucal }\end{array}$ \\
\hline Erva Lanceta & Solidago chilensis Meyen (Asteraceae) & $\begin{array}{l}\text { Anti-inflamatória, Uso em } \\
\text { Pancadas }\end{array}$ \\
\hline Arruda & Ruta graveolens L. (Rutaceae) & $\begin{array}{l}\text { Inseticida, Combate a } \\
\text { Ectoparasitas, Repelente }\end{array}$ \\
\hline Artemísia & Artemisia vulgaris L. (Asteraceae) & $\begin{array}{l}\text { Antiespasmódica Uterina, } \\
\text { Calmante }\end{array}$ \\
\hline Babosa & Aloe vera $(L)$ Burm.f. (Xanthorroeaceae) & $\begin{array}{l}\text { Emoliente, Cicatrizante, } \\
\text { Tratamento de } \\
\text { Hemorroidas, Cosmética }\end{array}$ \\
\hline Boldo Gambá & $\begin{array}{l}\text { Plectranthus neochilus Schltr. } \\
\text { (Lamiaceae) }\end{array}$ & $\begin{array}{c}\text { Distúrbios Estomacais, } \\
\text { Digestória }\end{array}$ \\
\hline Boldo Nacional & Plectranthus barbatus And. (Lamiaceae) & $\begin{array}{c}\text { Antiúlcera, Distúrbios } \\
\text { Estomacais, Ressaca, } \\
\text { Digestória }\end{array}$ \\
\hline Cana de Macaco & Costus spicatus (Jacq.) Sw. (Costaceae) & $\begin{array}{c}\text { Diurética, Sudorífera e } \\
\text { Tônica }\end{array}$ \\
\hline Capim-santo & $\begin{array}{c}\text { Cymbopogon citratus (DC.) Stapf. } \\
\text { (Poaceae) }\end{array}$ & $\begin{array}{c}\text { Ansiolítica, } \\
\text { Antiespasmódica }\end{array}$ \\
\hline Carqueja & $\begin{array}{c}\text { Baccharis trimera (Less.) DC } \\
\text { (Asteraceae) }\end{array}$ & $\begin{array}{l}\text { Distúrbios Digestórios, } \\
\text { Anti-inflamatória, } \\
\text { Desintoxicante Hepático }\end{array}$ \\
\hline Cavalinha & Equisetum hyemale L. (Equisetaceae) & Diurética \\
\hline Chambá & $\begin{array}{l}\text { Justicia pectoralis Jacq. } \\
\text { (Acanthaceae) }\end{array}$ & $\begin{array}{l}\text { Broncodilatadora, } \\
\text { Analgésica, Anti- } \\
\text { inflamatória }\end{array}$ \\
\hline Citronela & $\begin{array}{c}\text { Cymbopogon winterianus Jowitt } \\
\text { (Poaceae) }\end{array}$ & $\begin{array}{l}\text { Repelente de Insetos, } \\
\text { Aromatizante de Ambientes }\end{array}$ \\
\hline Colônia & $\begin{array}{c}\text { Alpinia zerumbet (Pers.) B.L. } \\
\text { Burtt \& R.M. Sm } \\
\text { (Zingiberaceae) }\end{array}$ & $\begin{array}{l}\text { Anti-hipertensiva, } \\
\text { Ansiolítica }\end{array}$ \\
\hline Confrei & $\begin{array}{c}\text { Symphytum officinale L. } \\
\text { (Boraginaceae) }\end{array}$ & $\begin{array}{l}\text { Cicatrizante e } \\
\text { Anti-inflamatória } \\
\text { de Uso Externo }\end{array}$ \\
\hline
\end{tabular}


Vittalle - Revista de Ciências da Saúde v. 30, n. 1 (2018) 182-191

\begin{tabular}{|c|c|c|}
\hline Erva-Cidreira & $\begin{array}{c}\text { Lippia alba (Mill) N. E. Brown } \\
\text { (Verbenaceae) }\end{array}$ & $\begin{array}{c}\text { Calmante, } \\
\text { Antiespasmódica }\end{array}$ \\
\hline Hortelã da Folha Graúda & $\begin{array}{c}\text { Plectranthus } \\
\text { amboinicus (Lour.) Spreng. } \\
\text { (Lamiaceae) }\end{array}$ & $\begin{array}{c}\text { Broncodilatador, } \\
\text { Antitussígeno, Tratamento } \\
\text { da Asma }\end{array}$ \\
\hline Hortelã da Folha Miúda & $\begin{array}{c}\text { Mentha } x \text { villosa Huds } \\
\text { (Lamiaceae) }\end{array}$ & $\begin{array}{c}\text { Amebíase, Giardíase, } \\
\text { Carminativa }\end{array}$ \\
\hline Hortelã Japonesa & Mentha arvensis L. (Lamiaceae) & $\begin{array}{l}\text { Antitussígena, Antigripal, } \\
\text { Antidispéptica, Flavorizante }\end{array}$ \\
\hline Insulina Vegetal & $\begin{array}{c}\text { Cissus verticillata (L.) } \\
\text { Nicolson \& C.E.Jarvis (sin. } \\
\text { Cissus sicyoides L.) (Vitaceae) }\end{array}$ & $\begin{array}{l}\text { Hipoglicemiante, } \\
\text { Antidiabética }\end{array}$ \\
\hline Manjericão & $\begin{array}{l}\text { Ocimum basilicum } \mathrm{L} . \\
\text { (Lamiaceae) }\end{array}$ & $\begin{array}{l}\text { Condimentar, } \\
\text { Digestória, } \\
\text { Antisséptica das } \\
\text { Vias Aéreas }\end{array}$ \\
\hline Melão de São Caetano & $\begin{array}{c}\text { Momordica charantia L. } \\
\text { (Cucurbitaceae) }\end{array}$ & $\begin{array}{l}\text { Contra } \\
\text { Ectoparasitas, } \\
\text { Sarnicida }\end{array}$ \\
\hline Melissa & $\begin{array}{l}\text { Melissa officinalis L. } \\
\text { (Lamiaceae) }\end{array}$ & Calmante \\
\hline Neem & $\begin{array}{c}\text { Azadirachta indica A. Juss } \\
\text { (Meliaceae) }\end{array}$ & Repelente \\
\hline Poejo & $\begin{array}{l}\text { Mentha pulegium } \mathrm{L} . \\
\text { (Lamiaceae) }\end{array}$ & $\begin{array}{c}\text { Antisséptico das Vias } \\
\text { Aéreas }\end{array}$ \\
\hline Roma & $\begin{array}{l}\text { Punica granatum L. } \\
\text { (Punicaceae) }\end{array}$ & $\begin{array}{c}\text { Inflamações na Boca e na } \\
\text { Garganta }\end{array}$ \\
\hline Terramicina/ Sanativo & $\begin{array}{c}\text { Alternanthera brasiliana (L.) } \\
\text { Kunt (Amaranthaceae) }\end{array}$ & $\begin{array}{l}\text { Antisséptica para } \\
\text { Ferimentos }\end{array}$ \\
\hline Transagem & $\begin{array}{l}\text { Plantago major L. } \\
\text { (Plantaginaceae) }\end{array}$ & $\begin{array}{c}\text { Afecções Bucais, } \\
\text { Uso na Prisão de } \\
\text { Ventre }\end{array}$ \\
\hline
\end{tabular}

A denominação "Farmácias Vivas" surgiu no ano de 1983, no estado do Ceará, a partir do trabalho acadêmico do Prof. Dr. Francisco José de Abreu Matos na Universidade Federal do Ceará. O projeto consiste em oferecer assistência social farmacêutica, baseada no emprego científico de plantas medicinais e produtos delas derivados desenvolvidos no Brasil. Essa iniciativa emergiu da medicina tradicional através de preparações caseiras e a proposta considera a produção de plantas medicinais em pequenos, porém numerosos campos de cultivo regionalizados. Para isso, essas plantas devem ser cientificamente validadas com apoio técnico científico em termos de pessoal, material e bibliografia em um horto matriz, localizado junto às escolas de farmácia ou de saúde pública da região ou centros de ciências da saúde de universidades (13). Essa descrição do termo farmácias vivas se confunde com a própria definição de atividades de extensão acadêmica, quando articula os conhecimentos populares/tradicionais e científicos, a partir de uma demanda social e da atuação das universidades na formação profissional cidadã.

A extensão universitária protagonizou a estruturação do espaço por meio de demandas históricas vastamente descritas na literatura, por meio de estudos etnobotânicos sobre o uso de plantas medicinais que indicam, dentre outros elementos, a ampla biodiversidade vegetal no Brasil e as influências étnicas na formação da população brasileira como responsáveis pela popularização do uso das plantas medicinais (1). O município em que o CAV/UFPE encontra-se inserido apresenta uma rede de atenção básica extensa, responsável por contribuir com a formação dos estudantes desta instituição. As observações dos estudantes, em campo conjuntamente com os projetos iniciais 
desenvolvidos, direcionaram as ações subsequentes através de demandas relacionadas à formação de Agentes Comunitários de Saúde, à Formação de Preceptores alocados nas Unidades Básicas de Saúde, às Ações direcionadas para comunitários/usuários e/ou curiosos sobre o uso de plantas medicinais, assim como a incorporação de espécies no horto para futuras pesquisas baseadas no uso popular apresentado pelas observações ocorridas através do desenvolvimento dos projetos.

Assim, as atividades desenvolvidas no Horto de Plantas Medicinais do Espaço Farmácia Viva avançaram no sentido da formação de estudantes, profissionais de saúde e comunidade, por intermédio da participação de diversos projetos institucionais operacionalizados e coordenados de maneira interdisciplinar por variados atores da Universidade Federal de Pernambuco e parceiros, conforme se pode observar no Quadro 2.

Quadro 2: Projetos Desenvolvidos no Horto de Plantas Medicinais do Espaço Farmácia Viva da Universidade Federal de Pernambuco, Centro Acadêmico de Vitória, Vitória de Santo Antão, Pernambuco, Brasil.

\begin{tabular}{|c|c|c|c|c|}
\hline Projeto & Descrição & Edital & Coordenação & Vigência \\
\hline $\begin{array}{l}\text { Farmácia Viva: } \\
\text { Cultivando Saúde }\end{array}$ & \begin{tabular}{|c} 
Construir canteiros de plantas \\
medicinais e condimentares \\
(nativas e não nativas), \\
enfatizando a troca de \\
experiências com populares, \\
visando adequar a Farmácia Viva \\
à realidade da população local \\
enquanto espaço de educação \\
permanente.
\end{tabular} & $\begin{array}{l}\text { Edital n06- } \\
\text { Programa de } \\
\text { Extensão } \\
\text { Universitária - } \\
\text { PROEXT 2009/ } \\
\text { Ministério da } \\
\text { Educação }\end{array}$ & $\begin{array}{l}\text { René Duarte } \\
\text { Martins }\end{array}$ & \begin{tabular}{|c|}
$20 / 09 / 2009 a$ \\
$20 / 11 / 2010$
\end{tabular} \\
\hline $\begin{array}{c}\text { Informativo Verde } \\
\text { Vida }\end{array}$ & \begin{tabular}{|} 
Estruturação de um informativo \\
com periodicidade bimestral \\
sobre Práticas Integrativas e \\
Complementares, com enfoque \\
sobre o uso de plantas \\
medicinais e condimentares na \\
perspectiva de informar sobre \\
promoção e educação em saúde.
\end{tabular} & $\begin{array}{l}\text { PROEXT-2011-BEX } \\
\text { INTERIORIZAÇÃO }\end{array}$ & $\begin{array}{l}\text { René Duarte } \\
\text { Martins }\end{array}$ & \begin{tabular}{|c|}
$03 / 03 / 2011 \mathrm{a}$ \\
$30 / 12 / 2013$
\end{tabular} \\
\hline $\begin{array}{c}\text { Programa Nacional } \\
\text { de Reorientação da } \\
\text { Formação } \\
\text { Profissional em } \\
\text { Saúde (Pró-Saúde) } \\
\text { articulado ao } \\
\text { Programa de } \\
\text { Educação pelo } \\
\text { Trabalho para a } \\
\text { Saúde (PET-Saúde) }\end{array}$ & $\begin{array}{c}\text { Promoção da saúde e prevenção } \\
\text { de doenças crônicas não } \\
\text { transmissíveis } \\
\text { (Subprojeto: Uso Racional de } \\
\text { Plantas Medicinais em Diabetes } \\
\text { e Hipertensão). }\end{array}$ & $\begin{array}{l}\text { PRO-Saúde / PET- } \\
\text { Saúde } 2012\end{array}$ & $\begin{array}{c}\text { Ana Wládia } \\
\text { Silva de Lima } \\
\text { Tutor } \\
\text { Subprojeto: } \\
\text { René Duarte } \\
\text { Martins }\end{array}$ & 2012 a 2014 \\
\hline $\begin{array}{c}\text { Curso de } \\
\text { Preceptoria em } \\
\text { Saúde }\end{array}$ & $\begin{array}{c}\text { Qualificar, em preceptoria } \\
\text { acadêmica, os profissionais de } \\
\text { saúde da Secretaria Municipal de } \\
\text { Saúde da Vitória de Santo Antão } \\
\text { que recebem, nos campos de } \\
\text { prática profissional, discentes de } \\
\text { graduação e da residência } \\
\text { multiprofissional do Centro } \\
\text { Acadêmico de Vitória de Santo } \\
\text { Antão. } \\
\text { Módulos: Plantas Medicinais I e } \\
\text { II }\end{array}$ & $\begin{array}{l}\text { PRO-Saúde / PET- } \\
\text { Saúde } 2012\end{array}$ & $\begin{array}{c}\text { Ana Wládia } \\
\text { Silva de Lima }\end{array}$ & 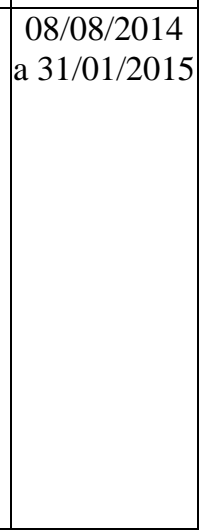 \\
\hline
\end{tabular}




\begin{tabular}{|c|c|c|c|c|}
\hline $\begin{array}{l}\text { Práticas Integrativas } \\
\text { e Complementares: } \\
\text { Mudança de } \\
\text { Paradigmas nas } \\
\text { UBS em Vitória de } \\
\text { Santo Antão. }\end{array}$ & $\begin{array}{l}\text { Desenvolver ações de ensino, } \\
\text { pesquisa e extensão no âmbito } \\
\text { das Práticas Integrativas e } \\
\text { Complementares, tendo como } \\
\text { objeto as Doenças Crônicas Não } \\
\text { Transmissíveis, analisando seus } \\
\text { resultados, acesso à atenção à } \\
\text { saúde e estruturação do Grupo de } \\
\text { Estudo e Trabalho em Práticas } \\
\text { Integrativas e Complementares } \\
\text { (GETPIC), no âmbito do Centro } \\
\text { Acadêmico de Vitória e } \\
\text { município da Vitória de Santo } \\
\text { Antão-PE. }\end{array}$ & $\begin{array}{l}\text { Proexc }- \text { Fluxo } \\
\text { Contínuo }\end{array}$ & \begin{tabular}{|c|} 
Rogélia \\
Herculano Pinto
\end{tabular} & \\
\hline $\begin{array}{c}\text { Plantas Medicinais } \\
\text { no cotidiano do } \\
\text { agente Comunitário } \\
\text { de Saúde: } \\
\text { valorizando a } \\
\text { cultura popular }\end{array}$ & $\begin{array}{c}\text { Capacitar ACS sobre o uso } \\
\text { correto de plantas medicinais e } \\
\text { fitoterápicos, disponibilizando } \\
\text { informações básicas sobre o } \\
\text { cultivo, indicações, manejo e } \\
\text { preparação de remédios caseiros } \\
\text { (xarope, pomada, xampu, elixir, } \\
\text { sabão entre outros) e } \\
\text { promovendo o intercâmbio e a } \\
\text { troca de experiências sobre o } \\
\text { papel dos Agentes Comunitários } \\
\text { de Saúde no uso correto de } \\
\text { plantas medicinais e fitoterápicos } \\
\text { na atenção básica de saúde. }\end{array}$ & $\begin{array}{c}\text { PROEXT-PIBEX } \\
2015\end{array}$ & \begin{tabular}{|l|} 
Danilo Augusto \\
Ferreira Fontes
\end{tabular} & \begin{tabular}{|c|}
$01 / 04 / 2015$ \\
a $31 / 01 / 2016$
\end{tabular} \\
\hline $\begin{array}{c}\text { Educação e Arte: } \\
\text { Saúde a toda parte, } \\
\text { ano III - Uso } \\
\text { Racional de Plantas } \\
\text { Medicinais: } \\
\text { Promoção da Saúde } \\
\text { e Preservação da } \\
\text { Biodiversidade }\end{array}$ & $\begin{array}{c}\text { Discutir sobre uso racional de } \\
\text { plantas medicinais, com enfoque } \\
\text { na preservação da biodiversidade } \\
\text { e promoção da saúde; }\end{array}$ & $\begin{array}{l}\text { PIBEX-Temático- } \\
2017\end{array}$ & $\begin{array}{c}\text { René Duarte } \\
\text { Martins }\end{array}$ & \begin{tabular}{|c|}
$01 / 04 / 2017$ \\
a $01 / 01 / 2018$
\end{tabular} \\
\hline
\end{tabular}

A indissociabilidade Ensino, Pesquisa e Extensão representa a base constitucional da formação universitária e deve ser equiparada com equidade de tratamento (14). Todavia, as dificuldades e as limitações presentes nas universidades para articular esses diferentes campos do saber acadêmico refletem na desarticulação entre demandas sociais, produção do conhecimento e formação universitária, muitas vezes contribuindo para a fragmentação entre os saberes populares, tradicionais e científicos $(15,16)$.

A flexibilização curricular pode contribuir com produção dialógica do conhecimento, ao proporcionar discussões entre docentes e discentes como campo pedagógico, por meio das vivências proporcionadas por atividades extensionistas (17). Nesse sentido, a Universidade Federal de Pernambuco resolve ajustar os projetos político-pedagógicos dos cursos de graduação com a finalidade de contribuir com essa dinâmica pedagógica ao Regulamentar a inserção e o registro de Ações Curriculares de Extensão (ACEx), como carga horária nos Projetos Pedagógicos de Cursos de Graduação da UFPE, em um mínimo de $10 \%$ da carga horária total dos cursos (18).

A necessidade de curricularizar as práticas emergentes dos projetos de extensão, com 
garantia da oportunidade de convívio com os saberes discutidos, para estudantes que não participavam dos projetos, suscitou a oferta de disciplinas eletivas. Em razão da oportunidade de discussão sobre novos perfis de projetos político-pedagógicos de cursos vigentes, as disciplinas eletivas condensaram-se em disciplinas obrigatórias para o curso de enfermagem e para um módulo no bacharelado em nutrição, assim como houve a inserção da disciplina de práticas integrativas da saúde ainda na nascente do projeto do bacharelado em saúde coletiva (Quadro 3).

A exploração de conhecimentos didáticos sobre fitoterápicos na formação de profissionais de saúde fornecem embasamento científico para atuação profissional, com destaque para atenção básica. Em estudo realizado na Universidade Estadual de Montes Claros - Minas Gerais -, foi observado que 70,8\% dos estudantes dos cursos de Enfermagem, Medicina e Odontologia mostraram-se favoráveis à inserção de conteúdos relacionados à fitoterapia na graduação, entretanto apenas 18,7\% conheciam a existência da Política Nacional de Práticas Integrativas e Complementares em Saúde PICS (19).

Para Christensen e Barros (2010) (20), a oferta de cursos informativos constitui importante estratégia para introdução do debate sobre as PICS nos cursos médicos, a ser acompanhada por intermédio da avaliação a respeito do campo de interesse, crenças, atitudes, desejo de aprofundamento sobre o assunto e modelo de oferta para novos componentes, respeitando os diferentes aspectos filosóficos e paradigmáticos. Assim, os cursos informativos norteariam as reflexões acerca de temas não previstos na matriz curricular, enquanto a abordagem formativa poderia ocorrer através de disciplinas específicas ou abordagem transversal a saberes e conteúdos discutidos em outras disciplinas correlatas, oportunizando o conhecimento sobre novas tecnologias médicas (20).

Quadro 3: Disciplinas Ofertadas no Centro Acadêmico de Vitória/UFPE que utilizam o Horto de Plantas Medicinais enquanto campo de Prática

\begin{tabular}{|c|c|c|c|c|c|}
\hline $\begin{array}{c}\text { Disciplina } \\
\text { (Descrição/Código) }\end{array}$ & Curso & $\begin{array}{l}\text { Carga Horária } \\
\text { (Horas }-\mathbf{h})\end{array}$ & $\begin{array}{c}\text { Vagas } \\
\text { (Número } \\
\text { de Pessoas) } \\
\end{array}$ & $\begin{array}{c}\text { Eletiva/ } \\
\text { Obrigatória }\end{array}$ & Semestre \\
\hline $\begin{array}{l}\text { Plantas Medicinais Da Coleta } \\
\text { À Terapêutica (ENFE0096) }\end{array}$ & \begin{tabular}{|c|} 
Bacharelado \\
em Enfermagem
\end{tabular} & $\begin{array}{c}\text { 45horas } \\
\text { (15h Teóricas e } \\
\text { 30h Práticas) }\end{array}$ & 30 & Eletiva & $\begin{array}{c}2010.2 \mathrm{e} \\
2011.1\end{array}$ \\
\hline $\begin{array}{l}\text { Saúde Coletiva E Etnias } \\
\text { Indígenas (SAUD0054) }\end{array}$ & $\begin{array}{l}\text { Bacharelado em } \\
\text { Saúde Coletiva }\end{array}$ & $\begin{array}{c}\text { 45horas } \\
\text { (15h Teóricas e } \\
\text { 30h Práticas) }\end{array}$ & 30 a 45 & Eletiva & $\begin{array}{c}2014.2- \\
\text { Atual }\end{array}$ \\
\hline $\begin{array}{c}\text { Práticas Integrativas E } \\
\text { Complementares Em Saúde } \\
\text { (NEN0052) }\end{array}$ & $\begin{array}{c}\text { Bacharelado em } \\
\text { Enfermagem }\end{array}$ & $\begin{array}{c}90 \text { horas } \\
\text { (30h Teóricas e } \\
\text { 60h Práticas) } \\
\end{array}$ & 30 & Obrigatória & $\begin{array}{l}2016.1- \\
\text { Atual }\end{array}$ \\
\hline $\begin{array}{c}\text { Práticas Integrativas Da Saúde } \\
\text { (SAUD0030) }\end{array}$ & $\begin{array}{l}\text { Bacharelado em } \\
\text { Saúde Coletiva }\end{array}$ & $\begin{array}{c}45 \text { horas } \\
\text { (15h Teóricas e } \\
\text { 30h Práticas) }\end{array}$ & 30 & Obrigatória & $\begin{array}{l}2015.1- \\
\text { Atual }\end{array}$ \\
\hline
\end{tabular}

\section{Conclusão}

O desafio em estruturar espaços informativos/formativos em fitoterapia deve recrutar esforços articulados entre docentes e instituição promotora, com envolvimento de captação de recursos, quando possível, além de parcerias com outros espaços de acúmulo intelectual e prático sobre o tema. Projetos de extensão e pesquisa somados a disciplinas eletivas e a espaços de debate suportam as reflexões iniciais sobre o tema, sempre que não exista previsão de disciplinas na matriz curricular dos cursos. $\mathrm{O}$ aprofundamento desse tripé acadêmico deverá se consolidar pelo atendimento às 


\section{Vittalle - Revista de Ciências da Saúde v. 30, n. 1 (2018) 182-191}

demandas sociais observadas, através da pesquisa e extensão, como também pela oferta de disciplinas obrigatórias na graduação e na pós-graduação, associadas a discussões transversais sobre fitoterapia em disciplinas dos eixos básico e profissional dos cursos de graduação.

\section{Agradecimentos}

Este trabalho desenvolveu-se com apoio do Ministério da Educação, por intermédio da Secretaria de Educação Superior, da PROEXC/UFPE e do Ministério da Saúde.

Conflitos de Interesse: Os autores declaram que não existem conflitos de interesse no trabalho realizado.

\section{Referências}

1. Firmo WCA, Menezes VJM, Passos CEC, Dias CN, ALVES LPL, Dias ICL, et al. Contexto histórico, uso popular e concepção científica sobre plantas medicinais. Cadernos de Pesquisa. 2012;18(Especial): 90-95.

2. Brasil. Ministério da Saúde. Práticas integrativas e complementares: plantas medicinais e fitoterapia na Atenção Básica. Secretaria de Atenção à Saúde. Departamento de Atenção Básica. Brasília: MS, 2012; pag. 29.

3. Brasil. Ministério da Saúde. Secretaria de Ciência, Tecnologia e Insumos Estratégicos. Departamento de Assistência Farmacêutica. Política nacional de plantas medicinais e fitoterápicos / Ministério da Saúde, Secretaria de Ciência, Tecnologia e Insumos Estratégicos, Departamento de Assistência Farmacêutica. - Brasília: Ministério da Saúde, 2006: 21-32.

4. Nunes, Fátima Rosane. Fitoterapia - Aplicações Curativas das Plantas Medicinais. Santa Maria: Farmácia e Laboratório Homeopático Cruz Vermelha; 2002.

5. Lopes JMDC, Link D. Implantação De um Horto Didático de Plantas Bioativas no Município de Tupanciretã. Revista Eletrônica em Gestão, Educação e Tecnologia Ambiental. 2011; 2(2): 225 250.

6. Carmo TN, Lucas FCA, Lobato GJM, Gurgel ESC. Plantas Medicinais e Ritualísticas Comercializadas na Feira da 25 de Setembro, Belém, Pará. Enciclopédia Biosfera, Centro Científico Conhecer - Goiânia. 2015; 11(21): 3440 - 3467.

7. SANTOS JAA, Santos ECB, Magnata SSLP, Garcia JE, Martins RD. Diagnóstico e educação em saúde no uso de plantas medicinais: relato de experiência. Rev. Ciênc. Ext. 2016; 12(4): 183-196.

8. IBGE Cidades. [atualizado em 2015; acessado em 05 de novembro 2017]. Disponível em: https://cidades.ibge.gov.br/brasil/pe/vitoria-de-santo-antao/panorama.

9. Brasil. Ministério da Educação - Secretaria de Ensino Superior (Resultado Edital n 06 - Programa de Extensão Universitária - PROEXT 2009). Acessado em 30 de Outubro de 2017. Disponível em: [http://portal.mec.gov.br/dmdocuments/resultado proext2009_2.pdf].

10. Raja RR. Medicinally Potential Plants of Labiatae (Lamiaceae) Family: An Overview. Research Journal of Medicinal Plants 2012; 6(3): 203-213.

11. Lima RK, Cardoso MG. Família Lamiaceae: Importantes Óleos Essenciais com Ação Biológica e Antioxidante. Revista Fitos 2006; 3: 14-24.

12. Gonçalves JS, Domingos DQ, Mansanares ME, Silva ER, Biondi M. A Família Lamiaceae no Complexo de Serras da Bocaina e de Carrancas, Minas Gerais. 64 Congresso Nacional de Botânica Belo Horizonte, 10-15 de Novembro de 2013.

13. Lorenzi H, Matos FJA. Plantas medicinais no Brasil: nativas e exóticas cultivadas. Nova Odessa, SP: Instituto Plantarum, 2002, $512 \mathrm{p}$.

14. Moita FMGS, Andrade FCB. Ensino-pesquisa-extensão: um exercício de indissociabilidade na pósgraduação. Revista Brasileira de Educação 2009; 14 (41): 269-293.

15. Silva MS, Vasconcelos SD. Extensão Universitária e Formação Profissional: Avaliação da Experiência das Ciências Biológicas na Universidade Federal de Pernambuco. Estudos em Avaliação Educacional 2006; 17(33): 119-136. 
16. Magnani I. Ensino, pesquisa, extensão e a nova tipologia do ensino superior brasileiro . In: Anais da 25 Reunião Anual da Anped, GT 11, Apresentação de trabalho, 2002.

17. Jezine E. As Práticas Curriculares e a Extensão Universitária. In: CONGRESSO BRASILEIRO DE EXTENSÃO UNIVERSITÁRIA, 2. Anais do $2^{\circ}$ Congresso Brasileiro de Extensão Universitária, Belo Horizonte. Setembro de 2004. Disponível em: <http://www.ufmg.br/congrext/Gestao/Gestao12.pdf> Acesso em: 01 de Novembro de 2017.

18. Universidade Federal de Pernambuco - Conselho Coordenador de Ensino, Pesquisa e Extensão. Resolução 09/2017. Aprovada nos dias 04 e 05 de Julho 2017. Disponível em: [https://www.ufpe.br/documents/398575/400862/Res+2017+09+CCEPE.pdf/ 1409f7c3-cbe4-405bb541-f2522f2a9d79]. Acessada em 12 de Novembro de 2017.

19. Feitosa MHA, Soares LL, Borges GA, Andrade MM, Costa SM. Inserção do Conteúdo Fitoterapia em Cursos da Área de Saúde. Revista Brasileira de Educação Médica 2016; 40 (2): 197-203.

20. Christensen MC, Barros NF. Revista Brasileira de Educação Medica 2010; 34 (1): 97-105 . 